\title{
Article \\ Origin, phylogeny, variability and epitope conservation of SARS-CoV-2 worldwide
}

\author{
Filipa F. Vale ${ }^{1, *}$, Jorge M.B. Vítor ${ }^{1}$, Andreia T. Marques ${ }^{1}$, José Miguel Azevedo-Pereira ${ }^{2}$, Elsa Anes ${ }^{2}$ and Joao \\ Goncalves 2
}

1 Pathogen Genome Bioinformatics and Computational Biology, Research Institute for Medicines (iMedULisboa), Faculty of Pharmacy, Universidade de Lisboa, 1649-003 Lisboa, Portugal; f.vale@ff.ul.pt (F.F.V.); jvitor@ff.ulisboa.pt (J.M.B.V.); amarques@farm-id.pt (A.T.M.)

2 Host-Pathogen Interactions Unit, Research Institute for Medicines (iMed-ULisboa), Faculty of Pharmacy, Universidade de Lisboa, 1649-003 Lisboa, Portugal; miguel.pereira@ff.ul.pt (J.M.A.-P.); eanes@ff.ulisboa.pt (E.A.)

3 Molecular Microbiology and Biotechnology Department, Research Institute for Medicines (iMed.ULisboa), Faculty of Pharmacy, Universidade de Lisboa, Av. Prof. Gama Pinto, 1649-003, Lisbon, Portugal; jgoncalv@ff.ulisboa.pt (J.G.)

* Correspondence: vale.filipa@gmail.com or f.vale@ff.ul.pt

\begin{abstract}
The coronavirus disease 2019 (COVID-19) pandemic caused by the severe acute respiratory syndrome coronavirus 2 (SARS-CoV-2) challenges include understanding what triggered SARS-CoV-2 emergence, how this RNA virus is evolving or how the genomic variability may impact the primary structure of proteins that are targets for vaccine. We analyzed 19471 SARSCoV-2 genomes and 199,984 spike glycoprotein sequences available at the GISAID database from all over the world and 3335 genomes of other Coronoviridae family members available at Genbank, collecting SARS-CoV-2 high-quality genomes and distinct Coronoviridae family genomes. Here, we identify a SARS-CoV-2 emerging cluster containing 13 closely related genomes isolated from bat and pangolin that showed evidence of recombination, which may have contributed to the emergence of SARS-CoV-2. The analyzed SARS-CoV-2 genomes presented 9632 single nucleotide polymorphisms (SNPs) corresponding to a variant density of 0.3 over the genome, and a clear geographic distribution. SNPs are unevenly distributed throughout the genome and hotspots for mutations were found for the spike gene and ORF 1ab. We describe a set of predicted spike protein epitopes whose variability is negligible. All predicted epitopes for the structural E, $\mathrm{M}$ and $\mathrm{N}$ proteins are highly conserved. This result favors the continuous efficacy of the available vaccines.
\end{abstract}

Keywords: COVID-19, SARS-CoV-2 genomics, spike protein, epitope prediction, coronavirus comparative genomics

\section{Introduction}

The coronavirus disease 2019 (COVID-19) pandemic caused by the severe acute respiratory syndrome coronavirus 2 (SARS-CoV-2) rapidly spread throughout the world after an initial burst first reported in December 2019 at Wuhan, China, presumably after a host jump from animal to human [1-3].

Coronaviruses are non-segmented positive-sense single-stranded RNA viruses ranging from 26 to $32 \mathrm{~Kb}$ in length that belong to the family Coronaviridae, which is subdivided into four major genera: Alpha, Beta, Gamma and Delta-coronavirus [4]. Human coronaviruses were initially described in the 1960s associated with the common cold. There are seven coronaviruses that infect humans: two belong to the Alphacoronavirus genus and are responsible for non-severe disease (229E and NL63); the remaining five belong to the Betacoronavirus genus, two of them also causing mild, self-limited respiratory 
infections (OC43 and HKU1), and three associated with potentially lethal human respiratory infectious (SARS-CoV, MERS-CoV and SARS-CoV-2) [5]. While 229E, OC43, NL63, and HKU1 are well adapted to humans without an animal reservoir, SARS-CoV and MERS-CoV were not well adapted to humans in terms of transmission and have likely jumped from animal (bat, civet and camel) reservoirs [5]. Notably, SARS-CoV-2 has efficiently adapted to humans after a probable recent zoonotic event and is highly transmissible. Close contact with infecting animals provides the opportunity for a host jump, like the two recent epidemics by coronavirus, SARS-CoV (China) and MERS-CoV (Middle East) that had bats as reservoir species and that could be transmitted to humans also from secondary hosts or bridge species like civets and camels, respectively [5]. Indeed, bat SARS-related coronavirus presented sequence similarity and the same cell receptor as SARS-CoV-2, the angiotensin converting enzyme 2 (ACE2) [6]. The most probable scenarios for the origin of SARS-CoV-2 are those typical of a zoonosis and include natural selection in an animal reservoir host before zoonotic transfer, or natural selection in humans following zoonotic transfer, during undetected human-to-human transmission [7]. The bat and pangolin related coronavirus are the closest relative coronavirus to SARS-CoV-2 [8,9]. Namely, SARS-CoV-2 has high sequence identity with structural proteins of the recent isolated Malayan pangolin coronavirus, which led to the suggestion that pangolins may had been an intermediate host of SARS-CoV-2 $[9,10]$.

Importantly, there is a panoply of coronavirus able to infect a large variety of animals, including for instance livestock, exotic and companion animals and wildlife, allowing for the opportunity for genetic recombination resulting in novel viruses [5]. Additionally, the high mutation rate of RNA viruses, yielding offsprings that differ by 1-2 mutations from their parents [11], is correlated with enhanced virulence [12] and favors zoonotic events and epidemic spread, making RNA viruses such as Coronaviruses the most common found in new causes of human disease [13], like COVID-19. Accordingly, closely related coronavirus circulating in the wet animal markets or other places of close contact with humans may allow the cross-species spillover [6]. The high mutation rate also provides a means of escaping vaccine-induced immunity and treatment resistance [12]. Despite coronavirus encoding a proofreading exoribonuclease in the NSP14 gene that mediates high-fidelity RNA genome replication [14], the impact of the proof-reading in genome variability is not completely established. Thus, it is important to analyze the level of mutations in a large collection of genomes and evaluate their impact for the development of vaccine or diagnosis methods based on the detection of antibodies. For both, the spike gene is the major target, since the spike glycoprotein (S) is responsible for viral attachment and fusion with the host cell. The $S$ glycoprotein contains a receptor-binding domain (RBD) that specifically binds to ACE2 receptors, starting cell entry. Next, the cleavage of the $S$ glycoprotein by cellular proteases leads to fusion and endocytosis [15].

To combat the epidemic with a vaccine or with a drug it is vital to understand the genetic variability of SARS-CoV-2. Thus, the aim of the present work is to understand the probable origin of SARS-CoV-2 through sequence comparison with other coronavirus sequences available in public databases; and to contribute to the understanding of the variability of SARS-CoV-2 genomes and its impact on vaccines and diagnostic tests efficacy by analyzing nearly 20,000 SARS-CoV-2 high quality genomes and 200000 spike protein sequences available at GISAID database.

\section{Materials and Methods}

\subsection{Coronavirus genome sequences}

High coverage complete sequenced SARS-CoV-2 deposited at GISAID were retrieved for analysis, comprehending 19471 worldwide genome sequences. The SARS- 
CoV-2 NC_045512.2 (corresponding to reference EPI_ISL_402125 at GISAID database) was used as reference genome.

Genomes of Coronoviridae family available at NCBI were retrieved, totaling 3335 genomes. When available the natural host species was collected using an in-house Python script. Other human coronavirus genomes, including SARS-CoV (58 genomes), MERSCoV (599 genomes), 229E (43 genomes), NL63 (82 genomes), HKU1 (48 genomes) and OC43 (178 genomes), were retrieved from NCBI, totaling 1013 genome sequences.

\subsection{Phylogenetic analysis and allele diversity of SARS-Cov-2}

The 19471 SARS-CoV-2 genomes were aligned with the reference genome using MAFFT version 7 [16] default options. Maximum-likelihood phylogenetic trees from alignments of nucleotide were produced using fasttreeMP [17]. To visualize and annotate produced trees the Interactive Tree Of Life (iTOL) v4 [18] was used. For better readability of the phylogenetic tree and to reduce their complexity by eliminating leaves that contribute the least to the tree diversity a smaller dataset with a more even representation of the different phylogenetic groups was obtain after pruning the tree with Treemmer v0.2 [19], using the options -mc 100, to protect from pruning 100 genomes from each continent, keeping 1000 representative leafs. A similar tree pruning with the option -mc 10 to protect 10 genomes from each continent was used to select 100 representative genomes from the large phylogenetic tree. These genomes were used for a comparative genomic analysis with other coronaviruses.

SNPs were extracted from multiple alignments using SNP-sites [20] producing a vcf file which was processed by the vcftools suite [21] to determine the allele frequency from SARS-CoV-2 genomes. A plot of variant density was produced to show how many SNPs there are and how they are distributed along the genome using a Python script (available at http://alimanfoo.github.io/2016/06/10/scikit-allel-tour.html).

\subsection{Comparative genomics and genomic diversity among Coronoviridae and human} coronavirus

The 100 SARS-CoV-2 representative genomes and 3335 genomes from Coronoviridae family members were aligned using MAFFT version 7 [16]. A phylogenetic tree was produced from the nucleotide alignments using fasttreeMP [17] and was visualized with iTOL v4 [18], as described above. A phylogenetic network was also build using the Neighbor Net algorithm [22] implemented in the software SplitsTree 4.10 [23], which is a powerful tool for visualization conflicting and consistent information present in a dataset. The filter taxa option was applied to show only the reference genome of SARS-CoV-2, a genome of each human coronavirus, as well as coronavirus infecting other species clustering with the SARS-CoV-2 reference genome, to evaluate origin and potential relationships between them.

Each group of genomes of human coronavirus retrieved from NCBI (229E, HKU1, MERS-CoV, NL63, OC43, SARS-CoV) was aligned using MAFFT version 7 [16], a tree was produced and pruned so that 30 representative genomes of each group could be selected. The 100 representative genomes of SARS-CoV-2 and 30 genomes of each group of human coronavirus were aligned and a network was produce using SplitsTree4 [23], since networks may generate more effective presentations of intraspecific evolution. Indeed, a phylogenetic networks allows to observe reticulate events like hybridization, horizontal gene transfer, recombination, or gene duplication and loss [22,23].

Additionally, the phylogenetic tree of 100 SARS-Cov-2 plus 3335 genomes from Coronoviridae family allowed to retrieve the group B coronavirus genomes that cluster with SARS-CoV-2. This group of 15 genomes plus the 100 SARS-CoV-2 representative genomes are hereinafter referred to as SARS-Cov-2 emerging cluster. The SARS-CoV-2 emerging cluster was aligned using MAFFT version 7 [16], after which SNP-sites [20] and vcftools suite [21] was used as described above. The SARS-CoV-2 emerging cluster is formed by two subgroups, the SARS-CoV-2 genomes and the Betacoronavirus genus 
genomes that cluster with SARS-CoV-2. The genetic diversity between each of the groups was done using the PopGenome package [24] in R, namely determine Fsт (fixation index), which tests whether there is genetic structure in the population and quantifies the proportion of genetic variation that lies between subpopulations within the total population; nucleotide diversity to measure the degree of polymorphism in the two groups; and Tajima's $\mathrm{D}$ statistics to detect departures from neutrality. Additionally, a principal component analysis (PCA) was done using the $\mathrm{R}$ package adegenet [25]. Moreover, a similarity plot and a bootscan plot was build using Simplot v3.5.1 - program [26] using a window of 500 nucleotides, which was moved along the SARS-CoV-2 reference genome in steps of 50 nucleotides. This analysis allowed to evaluate possible recombination events in the SARSCoV-2 emerging cluster and the similarity of non-SARS-CoV-2 genomes to SARS-CoV-2 genome. The sliding window partitions along the alignment of the SARS-CoV-2 emerging cluster method involves the construction of bootstrapped neighbor joining trees. Recombination is detected when a SARS-CoV-2 genome jumps between different clusters in trees constructed from adjacent alignment partitions.

\subsection{Tracing epitope conservation of SARS-CoV-2 spike protein (S glycoprotein) and other} structural proteins

B-cell epitope prediction of the S glycoprotein (Accession number: YP_009724390.1) was done using BepiPred-2.0 [27] using default settings. For the 19471 SARS-CoV-2 genome sequences worldwide, the spike gene was extracted and translated using in-house Python scripts. The S glycoprotein sequences were aligned using MAFFT version 7 [16] and the positions of the identified epitopes with BepiPred-2.0 [27] with $>5$ amino acid residues in length were extracted with an in-house Python script. Next, a sequence logo graphical representation [28] of the amino acid residues multiple sequence alignment was created with WebLogo 3 [29]. A similar analysis was done for the other structural proteins of SARS-CoV-2, i.e., E (envelop protein, accession number: YP_009724392.1), M (membrane glycoprotein, accession number: YP_009724393.1) and N (nucleocapsid phosphoprotein, accession number: YP_009724397.2) proteins. Additionally, near 200000 S glycoprotein sequences available at GISAID were collected and the analysis was repeated to check if the conservation of amino acids hold. Thus, for $199984 \mathrm{~S}$ glycoprotein sequences (all greater than 1250 amino acids and from SARS-CoV-2 isolates from human hosts) the percent of conservation worldwide and by continent was determined for each of the predicted epitopes. For sequence logo determination a multiple alignment is needed, but performing an alignment of almost 200 thousand sequences can require huge computer power. Thus, we have previously selected $S$ glycoprotein unique sequences, then conduct the alignment using MAFFT version 7 [16] and then determining the sequence logos with WebLogo 3 [29]. Using PyMOL Molecular Graphics System, Version 2.0 [30] it was verified if the predicted epitopes are displayed at the surface of the proteins, whenever the 3D structure of the proteins was available in the protein data bank (PDB). The 3D structures with the accession numbers 6vxx, 5X29 and 6VYO for S glycoprotein, E protein and N phosphoprotein were used, respectively.

\section{Results}

\subsection{SARS-CoV-2 genome variability}

The Maximum-likelihood phylogenetic tree (Figure 1) of 19471 SARS-CoV-2 genomes created with fasttreeMP [17], demonstrates the presence of clades associated with the geographic area of isolation. American and European isolates make up the majority of these genomes. Highly similar genomes are shown collapsed in the phylogenetic tree (Figure 1), most probably representing isolates within the same transmission chain. Tree pruning helps visualization of these clusters after removing similar genomes that correspond to proximal tree nodes. Thus, the phylogenetic tree was pruned using Treemmer v0.2 [19] 
to increase its readability (Figure 2). Focusing on 1000 genomes in the pruned phylogenetic tree that represent worldwide diversity shows that each region contains multiple clades although for each world region there are dominant spreading clades. The existence of clades reveals a high genome variability, which is typical of RNA viruses, as evidenced by 9632 SNPs among the 19471 SARS-CoV-2 genomes. The variant density is around 0.3 over the genome, that is the raw data contains a SNP approximately every 3 bases of the genome, unevenly distributed along the genome (Figure 3, Table S1). Indeed, the variant density is higher for the first two mature peptides of orf1ab/orfa coding for the leader protein and nsp2, and from open reading frame 6 to 10. Importantly, although generally the spike glycoprotein presents a variant density of 0.31 , some of their conserved domains present a higher variant density, towards the N-terminal domain of the coronavirus spike glycoprotein that functions as a receptor binding domain (Table S1).

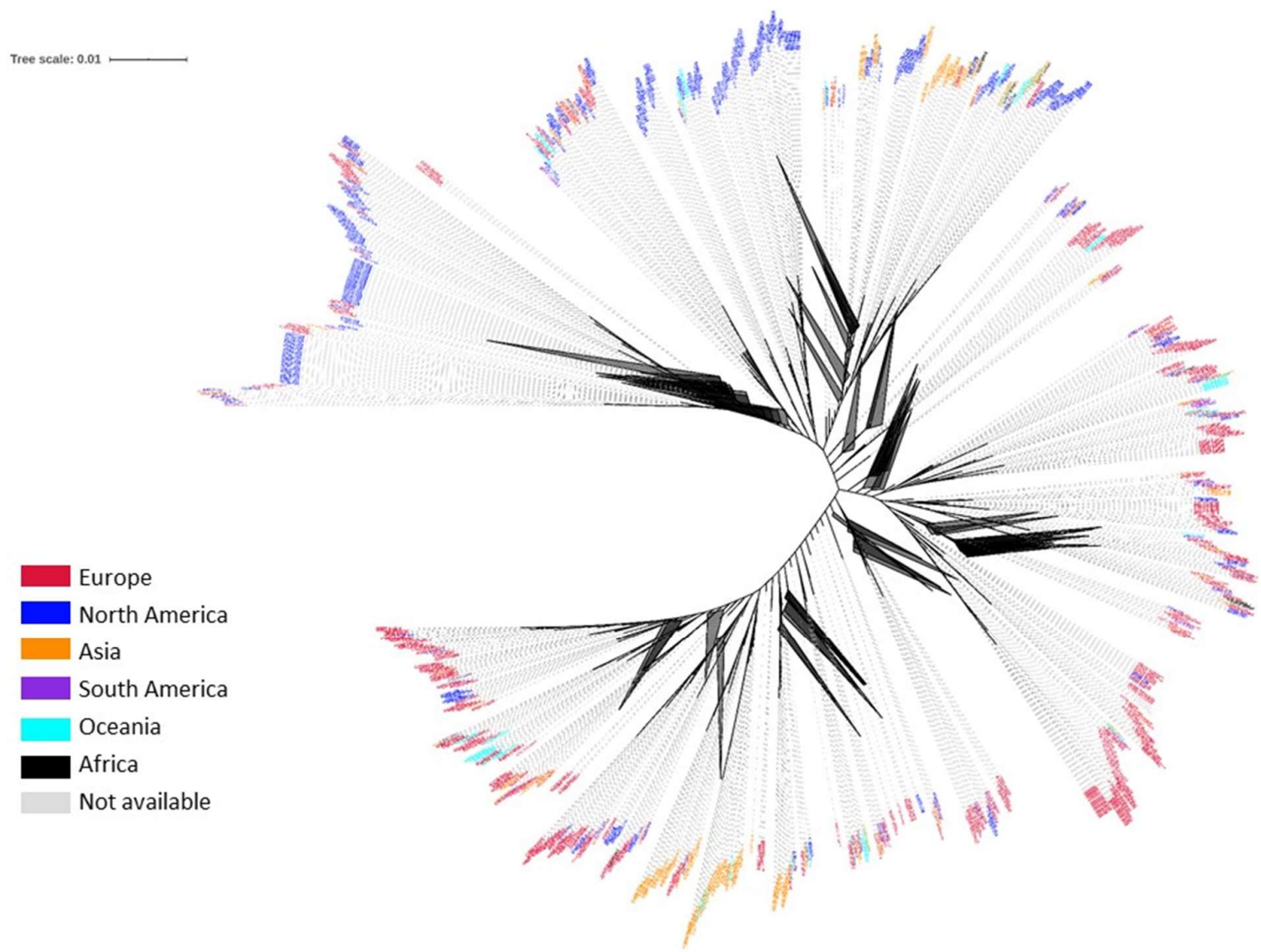

Figure 1. Maximum-likelihood phylogenetic tree of 19471 SARS-CoV-2 genomes, created with fasttreeMP and visualized with iTOL v4. Black triangles represent collapsed nodes of highly similar nodes (genomes). Each genome is colored coded by continent of origin. 


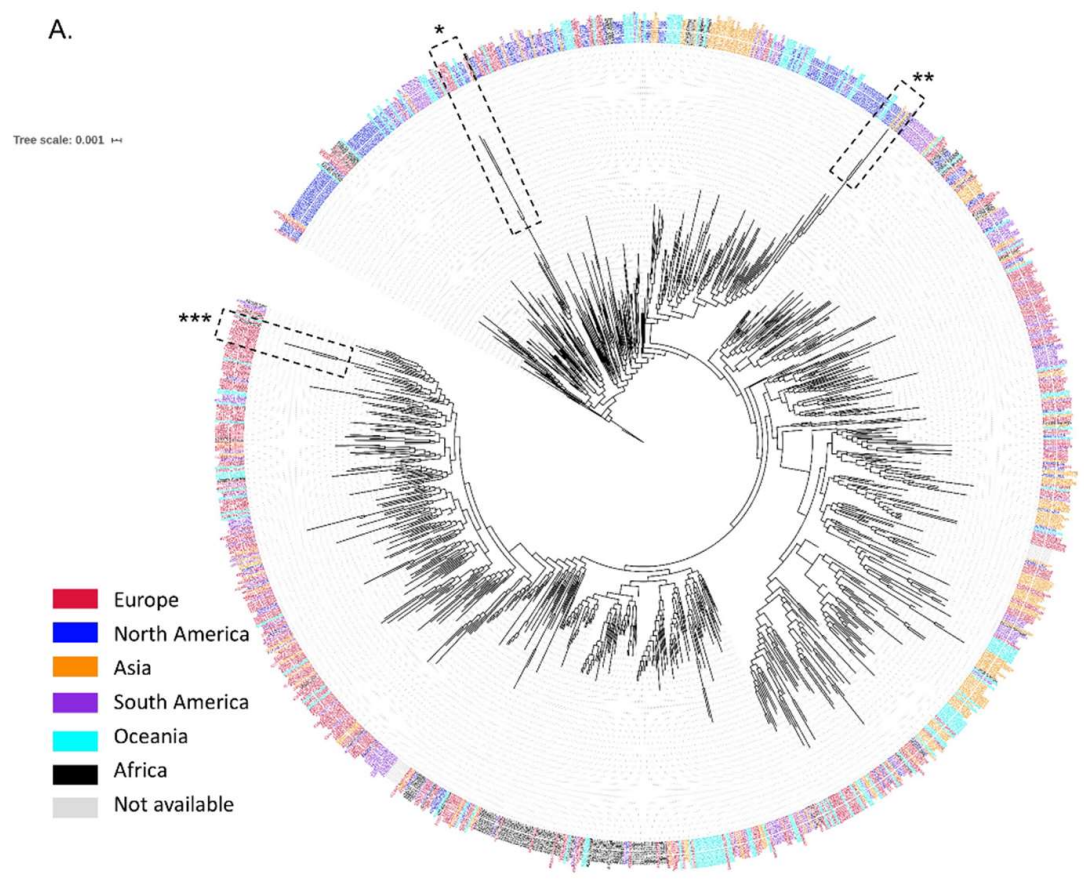

B.

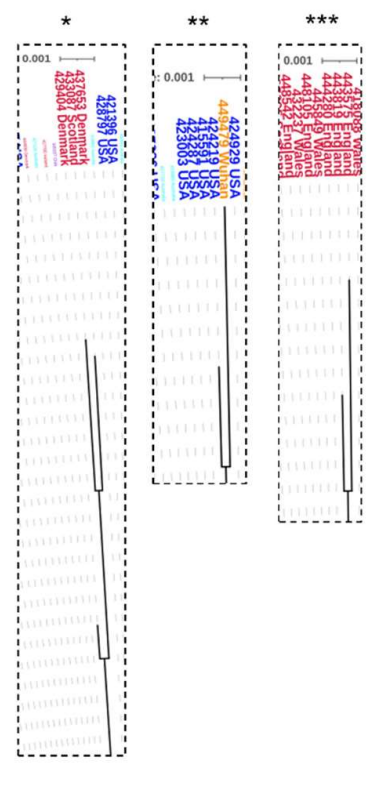

Figure 2. A) Trimmed phylogenetic tree of 1000 SARS-CoV-2 genomes representing the worldwide diversity, created with fasttreeMP and visualized with iTOL v4. Each genome is colored coded by continent of origin. B) Magnified detail view of topology of the phylogenetic tree evidencing strains in long branches.

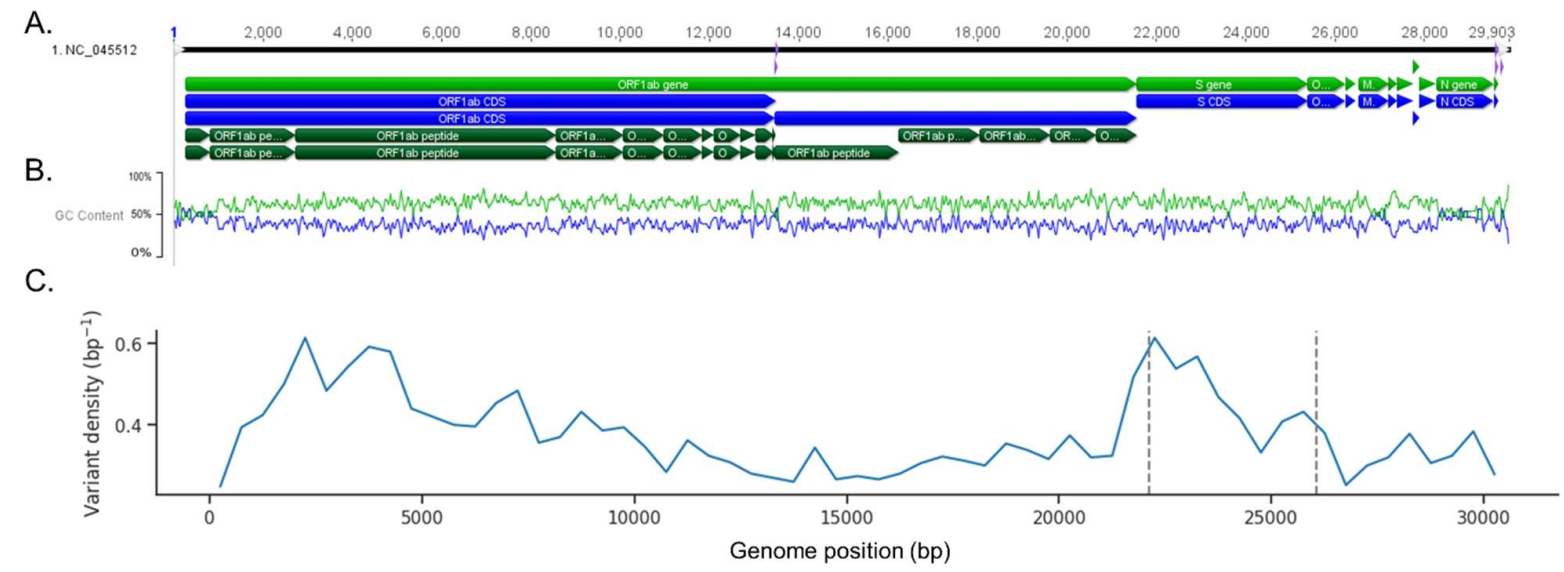

Figure 3. SNPs positions across SARS-CoV-2 genome. A) Genome map of SARS-CoV-2. B). GC content across the SARSCoV-2 genome. Blue - GC \% content; Green - AT \% content. C) Plot exhibiting SNPs distribution along the genome using a window size 500 bp; dashed grey bars indicate S gene position. (Figure caption using Geneious 8).

\subsection{Comparative genomics of SARS-CoV-2 and other Coronaviridae}

Tree pruning was also used to select 100 worldwide representative genomes from the large phylogenetic tree. Then, a comparison of a 100 representative SARS-CoV-2 genomes with 3335 genomes from other Coronoviridae family members showed, as expected, that the genomes cluster according to the coronavirus genera: Alpha, Beta, Delta and Gammacoronavirus and the more distant Toronovirinae subfamily (Figure 4). Coronavirus capable of infecting humans belong to distinct groups and those associated with milder disease outcomes are in Alphacoronavirus (229E-CoV and NL63-CoV), and Betacoronavirus (HKU1$\mathrm{CoV}$ and $\mathrm{OC} 43-\mathrm{CoV})$ genera. Betacoronavirus genus harbors all human coronavirus that have been provoking serious epidemic episodes (SARS-CoV, MERS-CoV and SARS-CoV- 
2) (Figure 4). A closer inspection of genomes clustering together with 100 representative SARS-CoV-2 genomes reveals 13 betacoronavirus genomes whose host is the bat or the pangolin (the remaining 2 genomes to complete the group of 115 correspond to SARSCoV-2 genomes retrieved from GenBank) (This detail of Figure 4 is zoom-in in Figure 5). The phylogenetic network analysis (Figure 6) presented using the filtering option of SplitsTree [23] to show only the SARS-CoV-2 reference genome and the 6 genomes of the bat and pangolin coronavirus that clusters together (the remaining 5 of the group of 13 coronavirus (Table 1 ) that cluster with the 100 representative SARS-CoV-2 genomes are similar to the ones showed above and for better readability are not presented) shows short inner branches and long terminal branch lengths leading to the tips, i.e. showing deep divergence between strain lineages. A similar observation occurs for the SARS-CoV genome from the 2003 epidemics and a close related bat genome (the bat coronavirus BM4831) (Figure 6). The inner reticulation branching pattern observable is indicative of extensive recombination [31]. However, while distinct human coronavirus are in different clusters, genomes of each group of human coronavirus cluster together (Figure S1).

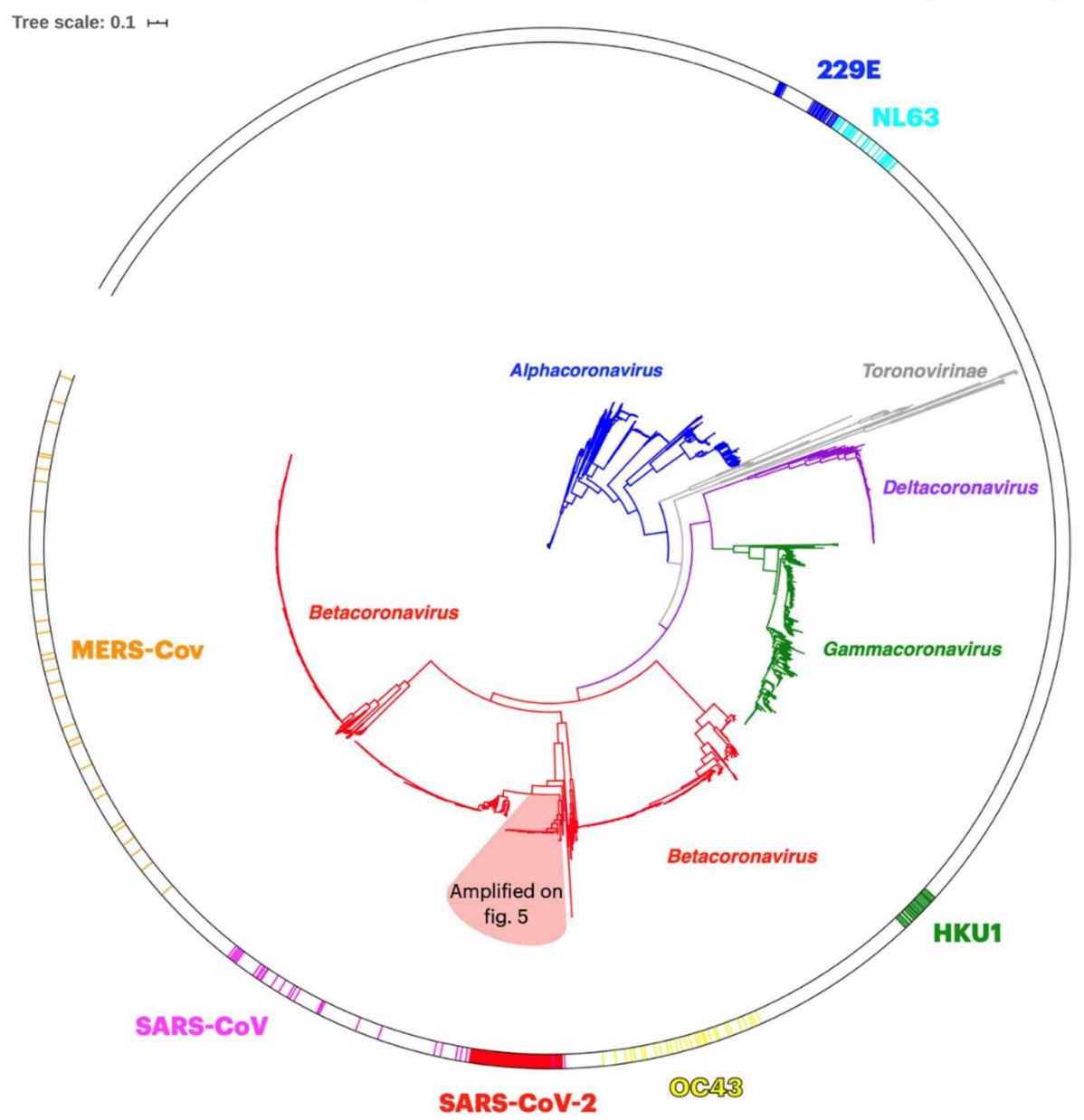

Figure 4. Maximum-likelihood phylogenetic tree of 3435 genomes of Coronaviridae family, created with fasttreeMP and visualized with iTOL v4. Blue clade - Alphacoronavirus; Grey clade - Toronovirinae subfamily; Violet clade - Deltacoronavirus; Green clade - Gammacoronavirus; Red clade - Betacoronavirus (includes 100 genomes of SARS-CoV-2 representing worldwide variability). Reddish cone represents the SARS-CoV-2 emerging cluster which is detailed in Figure 5 . The circular strip highlights coronavirus capable of infecting humans, colored clockwise as: Blue - 229E; Cyan - NL63; Green - HKU1; Yellow - OC43; Red - SARS-CoV-2 (detailed in Figure 5); Magenta - SARS-CoV; Orange - MERS-CoV. 


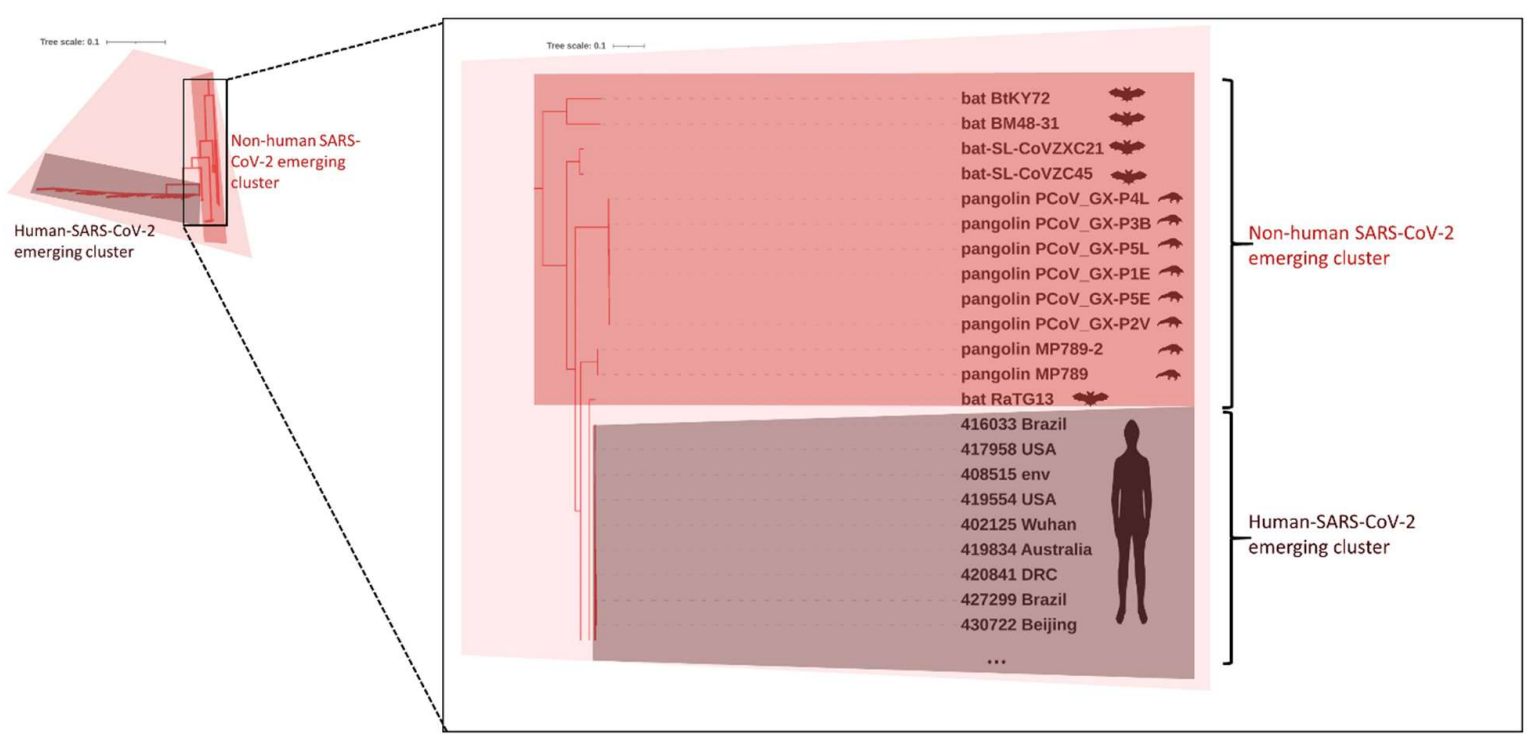

Figure 5. Magnified detail view of topology of the phylogenetic tree cluster from which SARS-CoV-2 emerged (corresponding to the reddish cone in Figure 4), evidencing all non-human SARS-CoV-2 emerging cluster and part of the human-SARS-CoV-2 emerging cluster. For SARS-CoV-2 genomes belonging to the human-SARS-CoV-2 emerging cluster just a few ones are showed to better readability (genome codes from GISAID identify the genomes). For other species the isolate name is presented. The host of the coronavirus is represented by human, bat and pangolin cartoons.

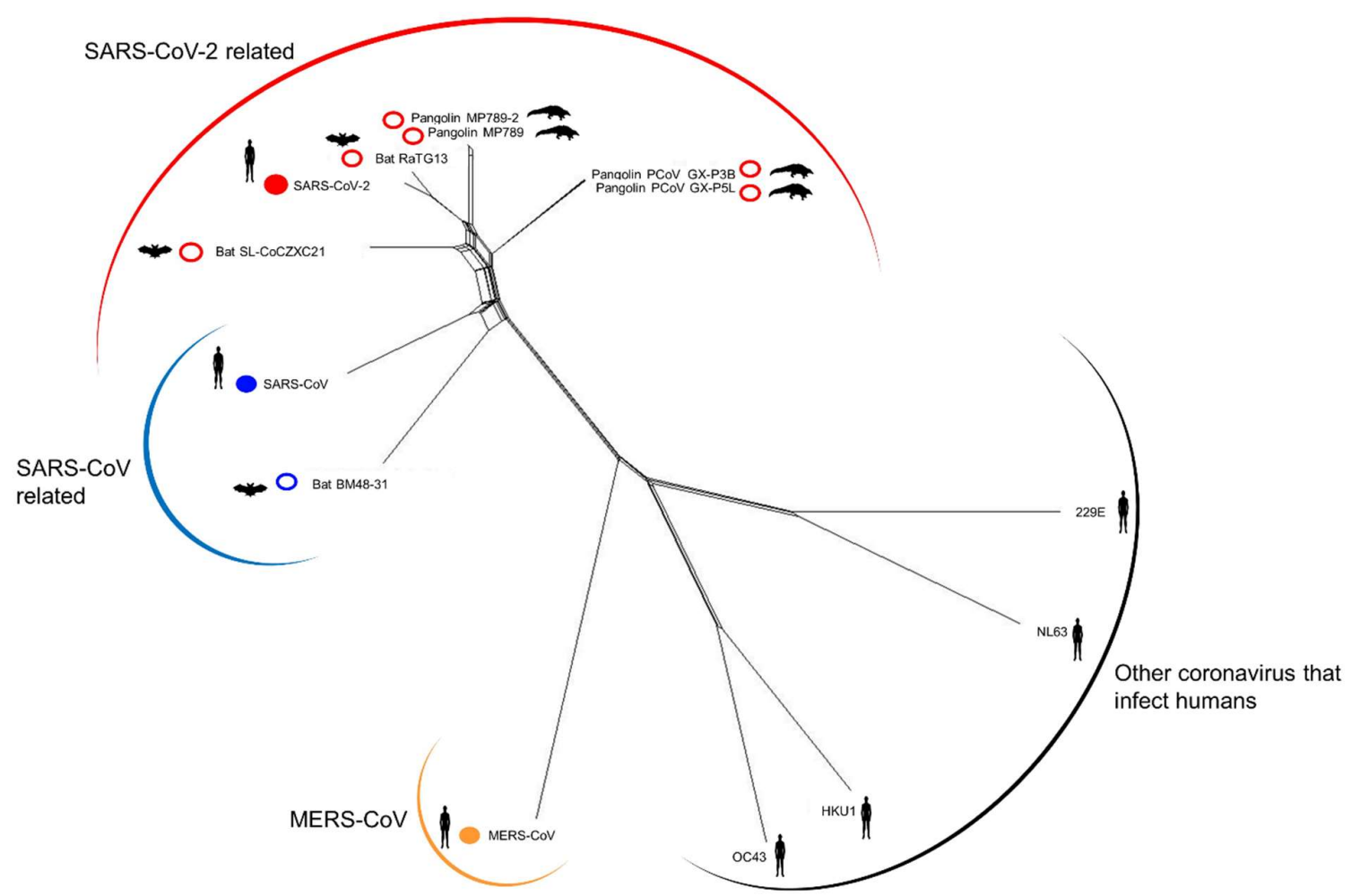

Figure 6. Filter of the SplitsTree network of the coronavirus family evidencing the reference SARS-CoV-2 genome (filled red circle) and other coronavirus belonging to the same cluster (unfilled red circle). SARS-CoV genome (filled blue circle) and a closer bat genome (unfilled blue circle) is showed. MERS-CoV genome is showed (filled orange circle) as well as one genome of each coronavirus group that infects humans. The host of the coronavirus is represented by human, bat and pangolin cartoons.

Table 1. Non-human-SARS-CoV-2 emerging cluster data. 


\begin{tabular}{|c|c|c|c|c|c|c|c|}
\hline Strain & Host & $\begin{array}{l}\text { Isolation } \\
\text { country }\end{array}$ & $\begin{array}{l}\text { Collection } \\
\text { date }\end{array}$ & $\begin{array}{l}\text { Publica- } \\
\text { tion date }\end{array}$ & $\begin{array}{l}\text { \% similarity } \\
\text { SARS-CoV- } \\
2\end{array}$ & $\begin{array}{l}\text { Accession } \\
\text { number }\end{array}$ & Reference \\
\hline RaTG13 & Rhinolophus affinis & China & 2013 & 2020 & 96.114 & MN996532.1 & [35] \\
\hline $\begin{array}{l}\text { bat-SL-Co- } \\
\text { VZXC21 }\end{array}$ & Rhinolophus sinicus bat & China & 2015 & 2018 & 87.410 & MG772934.1 & [49] \\
\hline bat-SL-CoVZC45 & Rhinolophus sinicus bat & China & 2015 & 2018 & 87.640 & MG772933.1 & [49] \\
\hline MP789-2 & SARS-CoV pangolin & China & 2019 & 2020 & 89.926 & MT121216.1 & [37] \\
\hline MP789 & Manis javanica & China & 2019 & 2020 & 78.523 & MT084071.1 & {$[37]$} \\
\hline PCoV_GX-P4L & $\begin{array}{l}\text { Manis javanica Malayan pango- } \\
\text { lin }\end{array}$ & China & 2017 & 2020 & 85.235 & MT040333.1 & {$[10]$} \\
\hline PCoV_GX-P3B & pangolin & China & 2017 & 2020 & 80.234 & MT072865.1 & {$[10]$} \\
\hline PCoV_GX-P5L & $\begin{array}{l}\text { Manis javanica Malayan pango- } \\
\text { lin }\end{array}$ & China & 2017 & 2020 & 85.245 & MT040335.1 & [10] \\
\hline PCoV_GX-P1E & $\begin{array}{l}\text { Manis javanica Malayan pango- } \\
\text { lin }\end{array}$ & China & 2017 & 2020 & 85.211 & MT040334.1 & {$[10]$} \\
\hline PCoV_GX-P5E & $\begin{array}{l}\text { Manis javanica Malayan pango- } \\
\text { lin }\end{array}$ & China & 2017 & 2020 & 85.208 & MT040336.1 & {$[10]$} \\
\hline PCoV_GX-P2V & pangolin & China & 2017 & 2020 & 85.211 & MT072864.1 & {$[10]$} \\
\hline BtKY72 & Rhinolophus sp. bat & Kenya & 2007 & 2019 & 74.654 & KY352407.1 & {$[50]$} \\
\hline $\begin{array}{l}\text { BM48- } \\
\text { 31/BGR/2008 }\end{array}$ & Rhinolophus blasii bat & Bulgaria & 2008 & 2010 & 74.638 & $\begin{array}{l}\text { NC_014470. } \\
1\end{array}$ & {$[51]$} \\
\hline
\end{tabular}

Note: This table just presents the subgroup of non-human-SARS-CoV-2 emerging cluster. The SARS-CoV-2 emerging cluster is composed of 115 genomes, of which 13 genomes belong to the non-human-SARS-CoV-2 emerging cluster and the remaining to the human-SARS-CoV-2 emerging cluster.

The SARS-CoV-2 emerging cluster is composed by 115 genomes that were separated in two subgroups, the non-SARS-CoV-2 genomes (13 genomes) and SARS-CoV-2 (102 genomes). These two subgroups are hereinafter referred to as non-human-SARS-CoV-2 emerging cluster and human-SARS-CoV-2 emerging cluster, respectively. The R PopGenome package [24] allowed to determine several statistics from multiple sequence alignments and single-nucleotide polymorphism (SNP) data of the SARS-CoV-2 emerging cluster. The Tajima-D statistics (a measure of the mutation frequency spectrum) in the sample for these subgroups was -2.894 and 0.070 , respectively. The negative value of this statistics reflects recent population expansion after a recent bottleneck, which is in agreement with the recent emerging of SARS-CoV-2 and rapid pandemic expansion. On the other hand, the roughly zero value points to a population with no evidence of selection. The nucleotide diversity was 3.810 and 1201.538, respectively, revealing that the degree of polymorphism in the non-human-SARS-CoV-2 emerging cluster subgroup was $>300$ superior than in the human-SARS-CoV-2 emerging cluster subgroup. FST value of 0.475 point to a differentiation among the two subgroups.

A principal component analysis (PCA), a technique for reducing the dimensionality of large datasets, was carry out in this group of 115 genomes done using the R package adegenet [25]. The first two principal components (Figure 7) explained $47.15 \%$ and $12.60 \%$ of the total variance in the dataset. The PCA analysis was able to divide the 115 genomes of the SARS-CoV-2 emerging cluster into three groups, namely SARS-CoV-2, a group of bat coronavirus genomes and a mixed group of bat and pangolin coronavirus genomes (Figure 7). In addition, the PCA confirms that the closest related genome to the SARSCoV-2 genomes corresponds to the bat coronavirus RaTG13 (Table 1). This distribution is also observable in the phylogenetic tree (Figures 5 and 7). The similarity plot performed with SimPlot [26] along the reference genome of SARS-CoV-2 shows how the genomes from the non-human-SARS-CoV-2 emerging cluster are related with the reference SARSCoV-2 genome (Figure S2.A). The recombination analysis, also performed with SimPlot [26], detected evidence of possible recombination (Figure S2.B), mainly around positions $\sim 2350$ to $\sim 2400$ (region of orf $1 \mathrm{ab}$ ) and 25400 to 25500 (region of spike gene) between the bat coronavirus RaTG13 and the group bat-SL-CoVZXC21-bat-SL-CoVZC45 and between the bat coronavirus RaTG13 and the pangolin coronavirus MP789, respectably (consult Table 1 for details). 

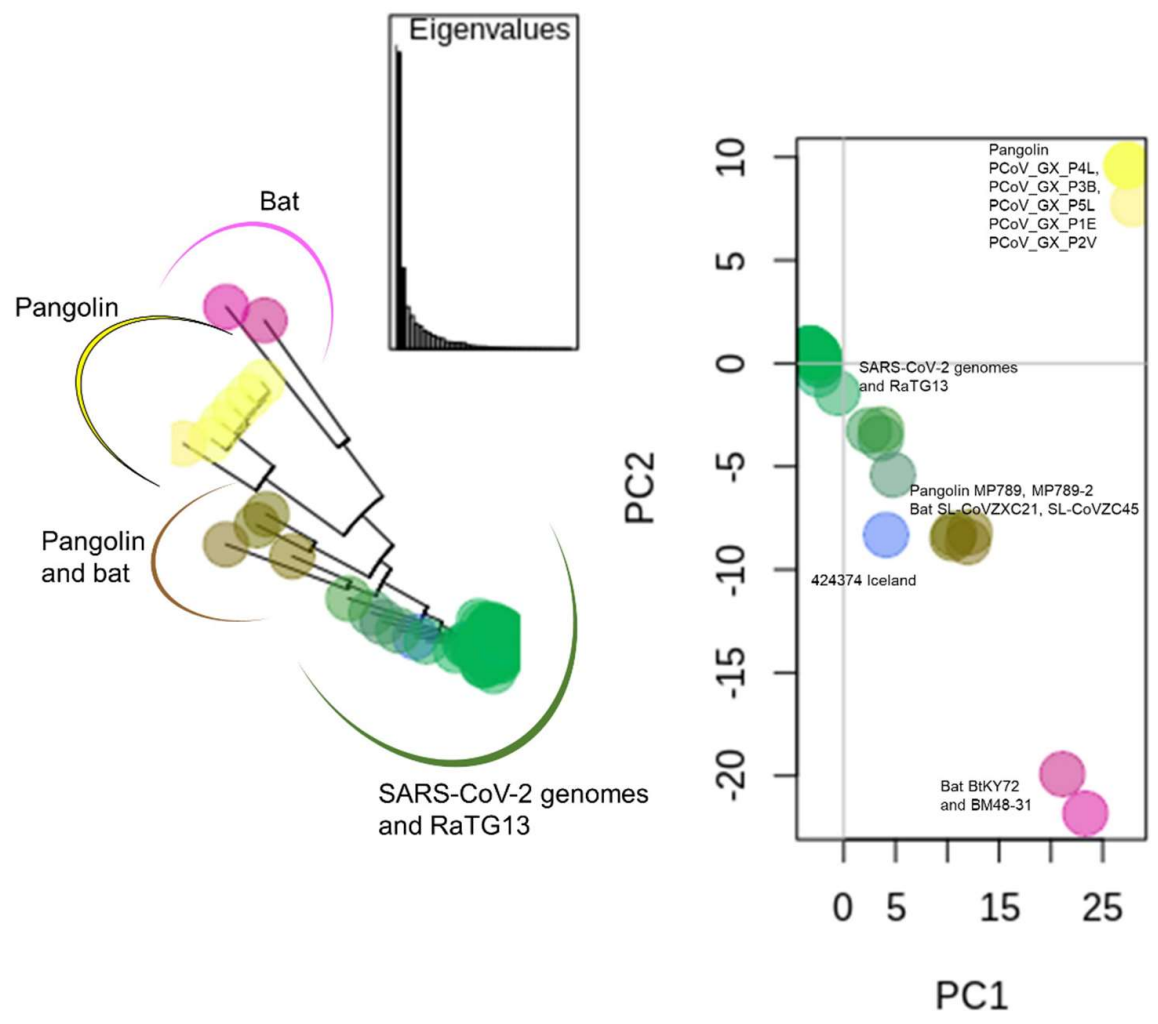

Figure 7. Phylogenetic tree and principal component analysis (PCA) of 115 genomes of the SARS-CoV-2 emerging cluster, done with R package adegenet. Phylogenetic tree exhibiting the same colors as in the scatter plot of the first two principal components. PC1 explains $47.15 \%$ of the variance and PC2 $12.60 \% \%$ of the variance. Green dots - SARS-CoV2 and the bat coronavirus RaTG13; Blue dot - SARS-CoV-2 genome (424374 Iceland); pink dots - bats Rhinolophus sp. (BtKY72) and Rhinolophus blasii (BM48-31); yellow dots - several pangolin Manis javanica; brown dots - pangolin (MP789-2 and MP789) and bat (Rhinolophus sinicus bat-SL-CoVZC45and bat-SL-CoVZXC21) genomes.

\subsection{Glycoprotein S - glycoprotein epitope conservation}

This study focused on predicted antibody-epitope interactions of the spike glycoprotein. B-cells play an important role in the adaptive immune system due to the production of antibodies that recognize target antigens by binding to a specific epitope in the antigen. Vaccines rely on the humoral immune response and attenuated or subunit vaccines that mimic the presentation of antigens to stimulate antibody production [27]. The SARS-CoV$2 \mathrm{~S}$ glycoprotein (a spike protein) is a good target for vaccine development: first, because of the role of this structural protein in viral attachment, fusion, and entry into the host cell [32]; secondly, because the generation of neutralizing antibodies to the spike protein should certainly block virus entry. Using the $S$ glycoprotein sequence of the SARS-CoV-2 genome, 29 epitopes with more than 5 amino acids were predicted, ranging from 6 to 26 amino acid residues (Figure S3, Table S2 Movies S1 and S2) using BepiPred-2.0, a sequence-based epitope prediction tool based on based on a random forest algorithm trained on epitope data from crystal structures, improving the algorithm predictive power [27]. Conserved epitopes are likely to provide broader protection across multiple virus strains, than those derived from highly variable genome regions. The degree of the predicted epitopes conservation was evaluated. The impact in epitope sequence conservation was residual. In fact, the 29 predicted epitopes appear to be conserved across the 19471 
SARS-CoV-2 genomes, as observed by the epitopes' sequence logos (Table S2). In a logo the height of the stack represents the sequence conservation at each position and the height of the amino acid symbol within the stack represents its relative frequency at that position [29]. The conservation of S glycoprotein predicted epitopes is high considering that the height of the stack is close to maximal for the majority of the residues and each stack has a clear predominant amino acid residue at every position in the epitope. Since the pdb 3D structure of the S glycoprotein is available (pdb: 6vxx), we have checked which of the predicted epitopes have their amino acids exposed in the 3D structure, verifying that about half are presumably entirely exposed (Table S2), favoring their application for vaccine development. Additionally, we have determined the percentage of conservation of the predicted epitopes among the 19471 SARS-CoV-2 S glycoprotein sequences. In agreement with the sequence logos, the predicted epitopes sequences were conserved between $92.6 \%$ and $95.6 \%$ of the S glycoprotein sequences (Table S2). In an unprecedented sequencing effort, new SARS-CoV-2 genomes are deposit at a daily basis at GISAID database. Thus, to verify the conservation of the $S$ glycoprotein predicted epitopes, we have repeated the analysis for 199984 spike glycoprotein sequences (Table S3). We found that the predicted epitopes maintained their high percentage of conservation worldwide (the predicted epitope sequence is found with $100 \%$ identity in a high percentage of analyzed sequences), varying from $85.7 \%$ to $99.8 \%$. The percentage of conservation determined by continent showed that the predicted epitopes are conserved across continents, with a punctual exception for one of the predicted epitopes in Oceania (Table S3). Of the 199984 S glycoprotein sequences, 31323 are unique sequences. This conservation is also observable in the sequence logos obtain from the multiple alignment of the 31323 S-glycoprotein unique sequences, where the sequence logo is almost always represented by a single amino acid (Table S2). Besides well conserved as observed by the sequences logos, in the $31323 \mathrm{~S}$ glycoprotein sequences the proportion of each unique sequence is not identical for all sequences. The most prevalent $S$ glycoprotein sequence is present in $37 \%$ (73997/199984) of the total S glycoprotein sequences, and the second and third most frequent sequences in $6.2 \%$ and $4.1 \%$, respectively.

\subsection{Other structural proteins (E, M and $N$ ) epitope conservation}

For the other three structural proteins of SARS-CoV-2, the E, M and N proteins the number of predicted epitopes using BepiPred-2.0 [27] with more than 5 amino acids was 1 (length of 15 amino acid residues, not predicted to be entirely exposed on the 3D structure of E protein), 4 (ranging from 6 to 21 amino acid residues) and 9 (ranging from 6 to 59 amino acid residues, half of each exposed at the 3D M protein surface), respectively (Tables S4, S5 and S6). In general, the predicted epitopes were found to have their sequences conserved in about $99 \%$ of the sequence for E, M and N proteins (Tables S4, S5 and S6). One of the predicted epitopes from the N phosphoprotein was found to be conserved only in about $70 \%$ of the sequences (Table S6).

\section{Discussion}

The emergence of the COVID-19 pandemic started a race to understand the origin of this outbreak and more importantly to plan strategies to overcome this global public health crisis. We applied phylogenetic and sequence analyses to address these pressing issues. The phylogenetic clustering is a powerful technique to understand how SARSCoV-2 genomes are related to each other and to other coronavirus that infect humans or animals, while sequence comparisons can identify which epitopes are stable versus those that are hotspots for mutation and are thus unsuitable as vaccine or diagnostic targets.

Applying phylogenetic analysis to 100 representative SARS-CoV-2 genomes plus 3335 genomes of other members of the Coronoviridae family demonstrated that they clustered into the 4 known Coronoviridae genera and the more distal Torovirinae genus. Focusing on the SARS-CoV-2 genomes confirmed their presence in the Betacoronavirus genus 
(Figure 4). Moreover, it identified a SARS-CoV-2 emerging cluster containing the 100 representative SARS-CoV-2 genomes and 13 genomes from bat and pangolin hosts (Figures 5 and 6). These findings suggest a likely link to viruses infecting these animal hosts.

The current knowledge on viral biodiversity is biased due to the limited number of closely related genomes available in public databases. The true betacoronavirus diversity is certainly far from being completely described, as databases represent mainly samples from human virus outbreaks [33], rather than non-human sources. This imposes a huge constraint and limitation in deciphering the origin of SARS-CoV-2. Continued sampling in areas where humans are in close contact with bats and pangolins may lead to the identification of closer relatives of SARS-CoV-2 [34]. Nonetheless, SARS-CoV-2 presents an average whole genome similarity of $96.1 \%$ with the bat virus RaTG13 strain isolated in China from Rhinolophus affinis (Table 1), making this genome the closest relative to SARSCoV-2 so far [35]. This observation is in agreement with bats being a significant reservoir for coronavirus from which spillovers infecting other species appear to routinely emerge [6]. In general, the non-human-SARS-CoV-2 emerging cluster presents less similarity to the reference SARS-CoV-2 genome in the regions coding for the ORF1ab polyprotein and the spike glycoprotein (Figure S2), which are precisely the regions of greatest variability among the SARS-CoV-2 genomes (Figure 3), as discussed below. Genetic recombination within positive-strand RNA viruses is an important evolutionary mechanism increasing viral diversity through the formation of novel chimeric genomes [36]. The present work showed evidence of recombination among the SARS-CoV-2 emerging cluster, which may have contributed to more efficient transmission and wider host range (Figures 2 and S2). Importantly, one of the regions where recombination was detected is precisely the spike gene, coding the $S$ glycoprotein responsible for initial attachment of the virus to the host cell [34]. The existence of an intermediate host, namely the pangolin, has been suggested $[8,10,37]$. This theory is supported by the observation that the E, M, N and S proteins of coronavirus isolated from pangolins showed $>90 \%$ amino acid identity and infected pangolins presented antibodies that reacted with the spike glycoprotein of SARS-CoV-2 [9]. The possible recombination detected between bat and pangolin coronavirus in the region of the spike glycoprotein, more specifically between bat genome RaTG13 isolated from Rhinolophus affinis and the pangolin genome MP789 isolated from Manis javanica, contribute to the theory that the pangolin was an intermediate host (Table 1). The PCA analysis confirmed the phylogenetic analysis of the SARS-CoV-2 emerging cluster, pointing to a genomic divergence from other betacoronaviruses. The related bat genome RaTG13 isolate in China from Rhinolophus affinis (Table 1) cluster together with SARS-CoV-2 genomes constituting a tight cluster, except for only one genome (from an Iceland SARS-CoV-2 isolate - GISAID EPI_ISL_424374). The Tajima D statistics may be computed either from within-species or among-species polymorphisms to test for neutrality [38]. The observed Tajima's D values $<0$ for SARS-CoV-2 is consistent with population expansion after a bottleneck, which is in agreement with others $[39,40]$. In opposition, the inter-species Tajima $\mathrm{D}$ near zero is compatible with absence of selection and neutral evolution. However, the nucleotide diversity among-species of the SARS-CoV-2 emerging cluster is an order of magnitude higher $(>300)$ than that observed within the species, which in combination with the recombination potential makes the SARS-CoV-2 emerging cluster a pool for potential emergence of novel coronavirus strains capable of infecting new hosts, like the SARSCoV-2.

When compared with other coronaviruses, SARS-CoV-2 forms a tight cluster. However, this does not mean that SARS-CoV-2 genomes are free of variation. On the contrary, when analyzing the world variability of nearly 20000 genomes a geographic distribution is clear, pointing to the ways of spread of the pandemic virus in each country and aggregating countries by continent. Interestingly, there are dominant virus spreading in each region (collapsed nodes of highly similar genomes, Figure 1). The current analysis is in agreement with others showing that the virus is evolving and that strains from different continents exhibit different mutation patterns [41,42]. The genomes collected from the GISAID database included isolates from the mint and tiger non-human hosts, and they 
cluster together with SARS-CoV-2, which points to a transmission from human to animal, demonstrating that SARS-CoV-2 has a host range larger than humans.

In the analyzed SARS-CoV-2 genomes one third of the genome has mutated in at least one of the analyzed genomes, totaling 9632 SNPs. However, these SNPs are not equally distributed along the SARS-CoV-2 genome and accumulate in hot spots for mutations, i.e., accumulating in the spike gene and ORF 1ab (Figure 3). These regions are precisely the ones showing detectable recombination (Figure S2) and where SARS-CoV-2 exhibits in general less similarity with the non-human-SARS-CoV-2 emerging cluster, suggesting that these genome regions are hypervariable. Most vaccines target the spike glycoprotein [43], because of the essential role of the $S$ protein in virus binding and uptake into the host cell allowing the replicative infection cycle to start. Certainly, the role of the spike protein in binding with host receptors makes it a perfect target for vaccine and antiviral therapeutic development [32]. The finding that the spike gene is a hotspot of variability in the SARS-CoV-2 genome might pose a problem for vaccine effectiveness as well as diagnostics and therapeutic targeting. However, a careful analysis of the impact of this variability in a set of predicted spike glycoprotein epitopes showed that presently this variability is negligible, which is a good predictor for the continuous success of a vaccine targeting the spike glycoprotein. Therefore, although the increased variability found for the spike gene (Figure 3.C.), this is not reflected in the amino-acids residues of the epitopes found (Table S2), which is in agreement with others [44]. The high degree of epitope conservation found in a large group of SARS-CoV-2 genomes confirms that this glycoprotein is a good target for vaccine development, especially if they rely on multiple epitope presentation. The conservation found for the epitopes may be related to the fact that most of the variants of the ACE2 human receptor are rare [45]. Even that a certain S protein presents for some epitope a sequence that differs from the epitope consensus sequence (Table S2), multiple epitope vaccines continue to stimulate the production of antibodies that still are capable of recognizing if not all at least some of the epitopes. Nonetheless, due to the fact that this is a hypervariable region a constant monitoring of the evolution of the sequence and its impact on epitope stability is mandatory. Accordingly, the predicted epitopes conservation analysis of the S glycoprotein for the 199984 sequences showed that they are worldwide conserved, keeping this conservation across each continent (Table S3) and over a time interval (Table S2). Even though most vaccines target the $S$ protein, other structural proteins have been proposed as vaccine targets, for being associated with viral envelope: $\mathrm{M}$ and $\mathrm{E}$; or for being highly immunogenic and abundantly expressed during infection by coronaviruses: $N$ protein $[43,46,47]$. The $S$ glycoprotein plays a crucial role in both viral replication and neutralization potential. The $\mathrm{E}$ protein has been associated with the pathogenesis of the cytokine storm observed in some patients with severe COVID-19 [48] and M protein has a major role in virion self-assembly. Furthermore, if these proteins are immunogenic and target for host antibodies, binding of these antibodies could block virus-cell interaction, precluding binding and/or fusion events through a mechanism of steric hindrance. We have thus succeeded to predict epitopes for $\mathrm{E}, \mathrm{M}$ and $\mathrm{N}$ proteins, which are less abundant than in the $\mathrm{S}$ glycoprotein (due to the smaller size of these proteins), but well conserved in nearly 20000 SARS-CoV-2 genomes (Tables S4, S5 and S6), supporting their application as vaccine targets.

In the current century this is the third emergency caused by coronavirus, and it is highly probable that new viruses will continue to emerge causing outbreaks due to their ability to mutate, recombine, and infect multiple species. The current study points to bats as the main reservoir of diversity of SARS-like coronaviruses, evidencing their ability to change their genomes which may in turn trigger the capacity of emerge in novel hosts and escape vaccine-induced. Indeed, the present analysis evidenced the existence of all these properties typical of RNA virus, namely existence of recombination events and high mutational rate in SARS-CoV-2, that accumulate in genome hotspots, for the time being without an impact in the conservation of epitope sequences. 
Supplementary Materials: The following are available online at www.mdpi.com/xxx/s1, Figure S1: SplitsTree network of coronavirus infecting humans using SplitsTree 4.1; Figure S2: Similarity plot and Bootscan plot along the reference genome of SARS-CoV-2 using SimPlot version3.5.1.; Figure S3: SARS-CoV-2 S glycoprotein structure (pdb: 6vxx). Table S1: Variant density across SARS-CoV-2 Open reading frames (ORFs); Table S2: Predicted epitopes from spike (S) glycoprotein of SARSCoV-2; Table S3: Conservation of the predicted epitopes from spike (S) glycoprotein of SARS-CoV2 worldwide and by continent; Table S4: Predicted epitopes from envelope (E) protein of SARSCoV-2; Table S5: Predicted epitopes from membrane (M) glycoprotein of SARS-CoV-2; Table S6. Predicted epitopes from nucleocapsid (N) phosphoprotein of SARS-CoV-2; , Video S1: SARS-CoV$2 \mathrm{~S}$ glycoprotein 3D structure (pdb: 6vxx) evidencing the exposed epitoperanging from the amino acid residues 207 to 222, produced with PyMol version 2.0; Video S2: SARS-CoV-2 S glycoprotein 3D structure (pdb: 6vxx) evidencing all completely exposed epitopes according to the 3D structure. For list of epitopes consult Table S2, produced with PyMol version 2.0; DataInfo: Accession numbers of the analyzed sequences and GISAID acknowledge table.

Author Contributions: Conceptualization, FFV, JV, EA, JMAP and JG; methodology, FFV and AM; software, FFV.; validation, JV, EA, JMAP and JG; formal analysis, FFV; writing - original draft preparation, FFV; writing - review and editing, FFV, AM, JV, EA, JMAP and JG; funding acquisition, JG. All authors have read and agreed to the published version of the manuscript.

Funding: This research was funded by national funds from FCT - Fundação para a Ciência e a Tecnologia, I.P., projects UIDB/04138/2020 and UIDP/04138/2020. Filipa F. Vale is financed by FCT through Assistant Researcher grant CEECIND/03023/2017.

Data Availability Statement: The data underlying this article are available in the article and in its online supplementary material files named DataInfo.pdf. The in-house python scripts are available at https://github.com/v888888/programing/releases/tag/scriptsGenomics

Acknowledgments: This work is based on SARS-CoV-2 genomes available in the GISAID database, so we would like to express our appreciation to all the researchers who contributed to the effort of sequencing and make genomes available. The authors would like to thank Francine Perler for valuable comments and suggestions.

Conflicts of Interest: The authors declare no conflict of interest. The funders had no role in the design of the study; in the collection, analyses, or interpretation of data; in the writing of the manuscript, or in the decision to publish the results.

\section{References}

1. Lai, C.-C.; Shih, T.-P.; Ko, W.-C.; Tang, H.-J.; Hsueh, P.-R. Severe acute respiratory syndrome coronavirus 2 (SARS-CoV-2) and coronavirus disease-2019 (COVID-19): The epidemic and the challenges. Int. J. Antimicrob. Agents 2020, 55, 105924, doi:10.1016/j.ijantimicag.2020.105924.

2. Nakagawa, S.; Miyazawa, T. Genome evolution of SARS-CoV-2 and its virological characteristics. Inflamm. Regen. 2020, 40, 17, doi:10.1186/s41232-020-00126-7.

3. Lu, R.; Zhao, X.; Li, J.; Niu, P.; Yang, B.; Wu, H.; Wang, W.; Song, H.; Huang, B.; Zhu, N.; et al. Genomic characterisation and epidemiology of 2019 novel coronavirus: implications for virus origins and receptor binding. Lancet (London, England) 2020, 395, 565-574, doi:10.1016/S0140-6736(20)30251-8.

4. Wang, H.; Li, X.; Li, T.; Zhang, S.; Wang, L.; Wu, X.; Liu, J. The genetic sequence, origin, and diagnosis of SARS-CoV-2. Eur. J. Clin. Microbiol. Infect. Dis. Off. Publ. Eur. Soc. Clin. Microbiol. 2020, 39, 1629-1635, doi:10.1007/s10096-020-03899-4.

5. Su, S.; Wong, G.; Shi, W.; Liu, J.; Lai, A.C.K.; Zhou, J.; Liu, W.; Bi, Y.; Gao, G.F. Epidemiology, Genetic Recombination, and Pathogenesis of Coronaviruses. Trends Microbiol. 2016, 24, 490-502, doi:10.1016/j.tim.2016.03.003.

6. Fehr, A.R.; Perlman, S. Coronaviruses: an overview of their replication and pathogenesis. Methods Mol. Biol. 2015, 1282, 1-23, doi:10.1007/978-1-4939-2438-7_1.

7. Andersen, K.G.; Rambaut, A.; Lipkin, W.I.; Holmes, E.C.; Garry, R.F. The proximal origin of SARSCoV-2. Nat. Med. 2020, 26, 450-452. 
8. Dos Santos Bezerra, R.; Valença, I.N.; de Cassia Ruy, P.; Ximenez, J.P.B.; da Silva Junior, W.A.; Covas, D.T.; Kashima, S.; Slavov, S.N. The novel coronavirus SARS-CoV-2: From a zoonotic infection to coronavirus disease 2019. J. Med. Virol. 2020, doi:10.1002/jmv.26072.

9. Xiao, K.; Zhai, J.; Feng, Y.; Zhou, N.; Zhang, X.; Zou, J.-J.; Li, N.; Guo, Y.; Li, X.; Shen, X.; et al. Isolation of SARS-CoV-2-related coronavirus from Malayan pangolins. Nature 2020, 583, 286-289, doi:10.1038/s41586-020-2313-X.

10. Lam, T.T.-Y.; Jia, N.; Zhang, Y.-W.; Shum, M.H.-H.; Jiang, J.-F.; Zhu, H.-C.; Tong, Y.-G.; Shi, Y.-X.; Ni, X.-B.; Liao, Y.-S.; et al. Identifying SARS-CoV-2-related coronaviruses in Malayan pangolins. Nature 2020, 583, 282-285, doi:10.1038/s41586-020-2169-0.

11. Vignuzzi, M.; Andino, R. Closing the gap: the challenges in converging theoretical, computational, experimental and real-life studies in virus evolution. Curr. Opin. Virol. 2012, 2, 515-518.

12. Duffy, S. Why are RNA virus mutation rates so damn high? PLoS Biol. 2018, 16, e3000003, doi:10.1371/journal.pbio.3000003.

13. Rosenberg, R. Detecting the emergence of novel, zoonotic viruses pathogenic to humans. Cell. Mol. Life Sci. 2015, 72, 1115-1125, doi:10.1007/s00018-014-1785-y.

14. Graepel, K.W.; Lu, X.; Case, J.B.; Sexton, N.R.; Smith, E.C.; Denison, M.R. Proofreading-Deficient Coronaviruses Adapt for Increased Fitness over Long-Term Passage without Reversion of Exoribonuclease-Inactivating Mutations. MBio 2017, 8, e01503-17, doi:10.1128/mBio.01503-17.

15. Pillay, T.S. Gene of the month: the 2019-nCoV/SARS-CoV-2 novel coronavirus spike protein. J. Clin. Pathol. 2020, 73, 366-369, doi:10.1136/jclinpath-2020-206658.

16. Katoh, K.; Standley, D.M. MAFFT multiple sequence alignment software version 7: improvements in performance and usability. Mol.Biol Evol. 2013, 30, 772-780.

17. Price, M.N.; Dehal, P.S.; Arkin, A.P. FastTree 2--approximately maximum-likelihood trees for large alignments. PLoS One 2010, 5, e9490, doi:10.1371/journal.pone.0009490.

18. Letunic, I.; Bork, P. Interactive Tree Of Life (iTOL) v4: recent updates and new developments. Nucleic Acids Res. 2019, 47, W256-W259, doi:10.1093/nar/gkz239.

19. Menardo, F.; Loiseau, C.; Brites, D.; Coscolla, M.; Gygli, S.M.; Rutaihwa, L.K.; Trauner, A.; Beisel, C.; Borrell, S.; Gagneux, S. Treemmer: a tool to reduce large phylogenetic datasets with minimal loss of diversity. BMC Bioinformatics 2018, 19, 164, doi:10.1186/s12859-018-2164-8.

20. Page, A.J.; Taylor, B.; Delaney, A.J.; Soares, J.; Seemann, T.; Keane, J.A.; Harris, S.R. SNP-sites: rapid efficient extraction of SNPs from multi-FASTA alignments. Microb. genomics 2016, 2, e000056, doi:10.1099/mgen.0.000056.

21. Danecek, P.; Auton, A.; Abecasis, G.; Albers, C.A.; Banks, E.; DePristo, M.A.; Handsaker, R.E.; Lunter, G.; Marth, G.T.; Sherry, S.T.; et al. The variant call format and VCFtools. Bioinformatics 2011, 27, 2156-2158, doi:10.1093/bioinformatics/btr330.

22. Bryant, D.; Moulton, V. Neighbor-net: an agglomerative method for the construction of phylogenetic networks. Mol.Biol Evol 2004, 21, 255-265.

23. Huson, D.H.; Bryant, D. Application of phylogenetic networks in evolutionary studies. Mol.Biol Evol. 2006, $23,254-267$.

24. Pfeifer, B.; Wittelsbürger, U.; Ramos-Onsins, S.E.; Lercher, M.J. PopGenome: an efficient Swiss army knife for population genomic analyses in R. Mol. Biol. Evol. 2014, 31, 1929-1936, doi:10.1093/molbev/msu136.

25. Jombart, T.; Ahmed, I. adegenet 1.3-1: new tools for the analysis of genome-wide SNP data. Bioinformatics 2011, 27, 3070-3071, doi:10.1093/bioinformatics/btr521.

26. Lole, K.S.; Bollinger, R.C.; Paranjape, R.S.; Gadkari, D.; Kulkarni, S.S.; Novak, N.G.; Ingersoll, R.; Sheppard, H.W.; Ray, S.C. Full-length human immunodeficiency virus type 1 genomes from subtype C-infected seroconverters in India, with evidence of intersubtype recombination. J. Virol. 1999, 73, 152-160, doi:10.1128/JVI.73.1.152-160.1999.

27. Jespersen, M.C.; Peters, B.; Nielsen, M.; Marcatili, P. BepiPred-2.0: improving sequence-based B-cell epitope prediction using conformational epitopes. Nucleic Acids Res. 2017, 45, W24-W29, doi:10.1093/nar/gkx346.

28. Schneider, T.D.; Stephens, R.M. Sequence logos: a new way to display consensus sequences. Nucleic Acids 
Res. 1990, 18, 6097-6100, doi:10.1093/nar/18.20.6097.

29. Crooks, G.E.; Hon, G.; Chandonia, J.-M.; Brenner, S.E. WebLogo: a sequence logo generator. Genome Res. 2004, 14, 1188-1190, doi:10.1101/gr.849004.

30. Schrodinger LLC The PyMOL Molecular Graphics System, Version 1.82015.

31. Lassalle, F.; Beale, M.A.; Bharucha, T.; Williams, C.A.; Williams, R.J.; Cudini, J.; Goldstein, R.; Haque, T.; Depledge, D.P.; Breuer, J. Whole genome sequencing of Herpes Simplex Virus 1 directly from human cerebrospinal fluid reveals selective constraints in neurotropic viruses. Virus Evol. 2020, 6, veaa012, doi:10.1093/ve/veaa012.

32. Samrat, S.K.; Tharappel, A.M.; Li, Z.; Li, H. Prospect of SARS-CoV-2 spike protein: Potential role in vaccine and therapeutic development. Virus Res. 2020, 288, 198141, doi:10.1016/j.virusres.2020.198141.

33. Kitchen, A.; Shackelton, L.A.; Holmes, E.C. Family level phylogenies reveal modes of macroevolution in RNA viruses. Proc. Natl. Acad. Sci. U. S. A. 2011, 108, 238-243, doi:10.1073/pnas.1011090108.

34. Zhang, Y.-Z.; Holmes, E.C. A Genomic Perspective on the Origin and Emergence of SARS-CoV-2. Cell 2020, 181, 223-227, doi:10.1016/j.cell.2020.03.035.

35. Zhou, P.; Yang, X.-L.; Wang, X.-G.; Hu, B.; Zhang, L.; Zhang, W.; Si, H.-R.; Zhu, Y.; Li, B.; Huang, C.-L.; et al. A pneumonia outbreak associated with a new coronavirus of probable bat origin. Nature 2020, 579, 270-273, doi:10.1038/s41586-020-2012-7.

36. Bentley, K.; Evans, D.J. Mechanisms and consequences of positive-strand RNA virus recombination. J. Gen. Virol. 2018, 99, 1345-1356, doi:10.1099/jgv.0.001142.

37. Liu, P.; Jiang, J.-Z.; Wan, X.-F.; Hua, Y.; Li, L.; Zhou, J.; Wang, X.; Hou, F.; Chen, J.; Zou, J.; et al. Are pangolins the intermediate host of the 2019 novel coronavirus (SARS-CoV-2)? PLoS Pathog. 2020, 16, e1008421, doi:10.1371/journal.ppat.1008421.

38. Bhatt, S.; Katzourakis, A.; Pybus, O.G. Detecting natural selection in RNA virus populations using sequence summary statistics. Infect. Genet. Evol. J. Mol. Epidemiol. Evol. Genet. Infect. Dis. 2010, 10, 421-430, doi:10.1016/j.meegid.2009.06.001.

39. Fang, B.; Liu, L.; Yu, X.; Li, X.; Ye, G.; Xu, J.; Zhang, L.; Zhan, F.; Liu, G.; Pan, T.; et al. Genome-wide data inferring the evolution and population demography of the novel pneumonia coronavirus (SARS-CoV-2). bioRxiv 2020, doi:10.1101/2020.03.04.976662.

40. Laskar, R.; Ali, S. Phylo-geo-network and haplogroup analysis of 611 novel Coronavirus (nCov-2019) genomes from India. bioRxiv 2020, doi:10.1101/2020.09.03.281774.

41. Pachetti, M.; Marini, B.; Benedetti, F.; Giudici, F.; Mauro, E.; Storici, P.; Masciovecchio, C.; Angeletti, S.; Ciccozzi, M.; Gallo, R.C.; et al. Emerging SARS-CoV-2 mutation hot spots include a novel RNA-dependent-RNA polymerase variant. J. Transl. Med. 2020, 18, 179, doi:10.1186/s12967-020-02344-6.

42. Forster, P.; Forster, L.; Renfrew, C.; Forster, M. Phylogenetic network analysis of SARS-CoV-2 genomes. Proc. Natl. Acad. Sci. 2020, 117, 9241-9243, doi:10.1073/pnas.2004999117.

43. Funk, C.D.; Laferrière, C.; Ardakani, A. A Snapshot of the Global Race for Vaccines Targeting SARSCoV-2 and the COVID-19 Pandemic. Front. Pharmacol. 2020, 11, 937, doi:10.3389/fphar.2020.00937.

44. Dearlove, B.; Lewitus, E.; Bai, H.; Li, Y.; Reeves, D.B.; Joyce, M.G.; Scott, P.T.; Amare, M.F.; Vasan, S.; Michael, N.L.; et al. A SARS-CoV-2 vaccine candidate would likely match all currently circulating variants. Proc. Natl. Acad. Sci. 2020, 117, 23652 LP - 23662, doi:10.1073/pnas.2008281117.

45. Fujikura, K.; Uesaka, K. Genetic variations in the human severe acute respiratory syndrome coronavirus receptor ACE2 and serine protease TMPRSS2. J. Clin. Pathol. 2020, jclinpath-2020-206867, doi:10.1136/jclinpath-2020-206867.

46. Dutta, N.K.; Mazumdar, K.; Gordy, J.T. The Nucleocapsid Protein of SARS-CoV-2: a Target for Vaccine Development. J. Virol. 2020, 94.

47. Florindo, H.F.; Kleiner, R.; Vaskovich-Koubi, D.; Acúrcio, R.C.; Carreira, B.; Yeini, E.; Tiram, G.; Liubomirski, Y.; Satchi-Fainaro, R. Immune-mediated approaches against COVID-19. Nat. Nanotechnol. 2020, 15, 
630-645, doi:10.1038/s41565-020-0732-3.

48. Schoeman, D.; Fielding, B.C. Is There a Link Between the Pathogenic Human Coronavirus Envelope Protein and Immunopathology? A Review of the Literature. Front. Microbiol. 2020, 11, 2086, doi:10.3389/fmicb.2020.02086.

49. Hu, D.; Zhu, C.; Ai, L.; He, T.; Wang, Y.; Ye, F.; Yang, L.; Ding, C.; Zhu, X.; Lv, R.; et al. Genomic characterization and infectivity of a novel SARS-like coronavirus in Chinese bats. Emerg. Microbes Infect. 2018, 7, 154, doi:10.1038/s41426-018-0155-5.

50. Tao, Y.; Tong, S. Complete Genome Sequence of a Severe Acute Respiratory Syndrome-Related Coronavirus from Kenyan Bats. Microbiol. Resour. Announc. 2019, 8, doi:10.1128/MRA.00548-19.

51. Drexler, J.F.; Gloza-Rausch, F.; Glende, J.; Corman, V.M.; Muth, D.; Goettsche, M.; Seebens, A.; Niedrig, M.; Pfefferle, S.; Yordanov, S.; et al. Genomic characterization of severe acute respiratory syndrome-related coronavirus in European bats and classification of coronaviruses based on partial RNA-dependent RNA polymerase gene sequences. J. Virol. 2010, 84, 11336-11349, doi:10.1128/JVI.00650-10. 